
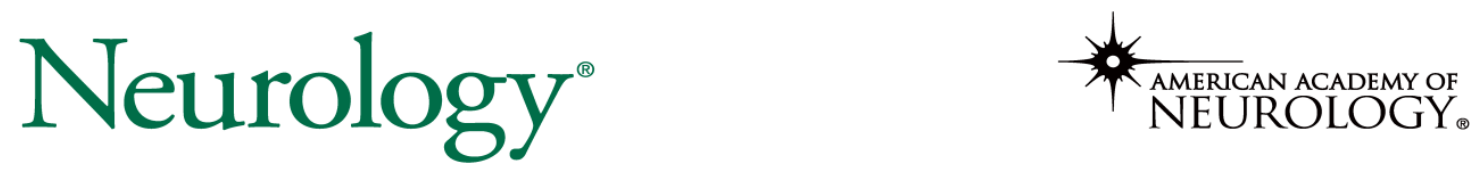

The most widely read and highly cited peer-reviewed neurology journal The Official Journal of the American Academy of Neurology

Neurology Publish Ahead of Print

DOI: 10.1212/WNL.0000000000013011

\title{
Teaching NeuroImage: Neurovascular Consequences of Autonomic Dysreflexia
}

Author(s):

Christina M Lineback, MD ${ }^{1}$; Eric W Moffet, MD ${ }^{1}$; Minjee Kim, MD ${ }^{1}$

\section{Corresponding Author:}

Christina M Lineback

christina.lineback@northwestern.edu

Neurology® Published Ahead of Print articles have been peer reviewed and accepted for publication. This manuscript will be published in its final form after copyediting, page composition, and review of proofs. Errors that could affect the content may be corrected during these processes. 
Affiliation Information for All Authors: 1. Department of Neurology, Northwestern University Feinberg School of Medicine, Chicago, IL

\section{Contributions:}

Christina M Lineback: Drafting/revision of the manuscript for content, including medical writing for content; Major role in the acquisition of data

Eric W Moffet: Drafting/revision of the manuscript for content, including medical writing for content Minjee Kim: Drafting/revision of the manuscript for content, including medical writing for content

Number of characters in title: 13

Abstract Word count: 100

Word count of main text: 100

References: 2

Figures: 2

Tables: 0

Neuroimage Legend Count: 56

Supplemental: 1. CARE 2. Patient consent3. Teaching Slides

Search Terms: [ 1 ] Autonomic diseases, [11 ] Stroke in young adults, [ 119 ] CT, [ 255 ] Spinal cord trauma; see Trauma/spinal cord trauma (S), [ 266 ] Spinal cord trauma

Study Funding: The authors report no targeted funding

Disclosures: The authors report no disclosures relevant to the manuscript. 
An 18-year-old man with C6 quadriparesis presented with two 30-minute episodes of thunderclap headache, vision loss, new urinary incontinence, hypertension (200s/90s), and bradycardia over 24 hours. Imaging demonstrated multifocal cerebrovascular narrowing (Figure 1), restricted diffusion and hyperintense T2/FLAIR signal (Figure 2); transcranial dopplers (TCDs) displayed increased velocities. Reversible cerebral vasoconstriction syndrome (RCVS) was diagnosed.

Neurogenic bladder perhaps triggered autonomic dysreflexia (AD) and thus RCVS.

Injury above $\mathrm{T} 6$ spinal cord eliminates supraspinal modulation, and can result in $\mathrm{AD}$, defined as episodic hypertension and bradycardia initiated by unrestrained sympathetic reflexes. ${ }^{1,2}$ After suprapubic catheter placement, episodes ceased, TCD velocities normalized, and vision returned. 
[AZ 10.8.2021] 173097 Teaching Slides -- http://links.lww.com/WNL/B638

\section{References}

1. Calabrese LH, Dodick DW, Schwedt TJ, Singhal AB. Narrative review: reversible cerebral vasoconstriction syndromes. Ann Intern Med. Jan 2007;146(1):34-44. doi:10.7326/00034819-146-1-200701020-00007

2. Ducros A. Reversible cerebral vasoconstriction syndrome. Lancet Neurol. Oct 2012;11(10):906-17. doi:10.1016/S1474-4422(12)70135-7

Figure 1:
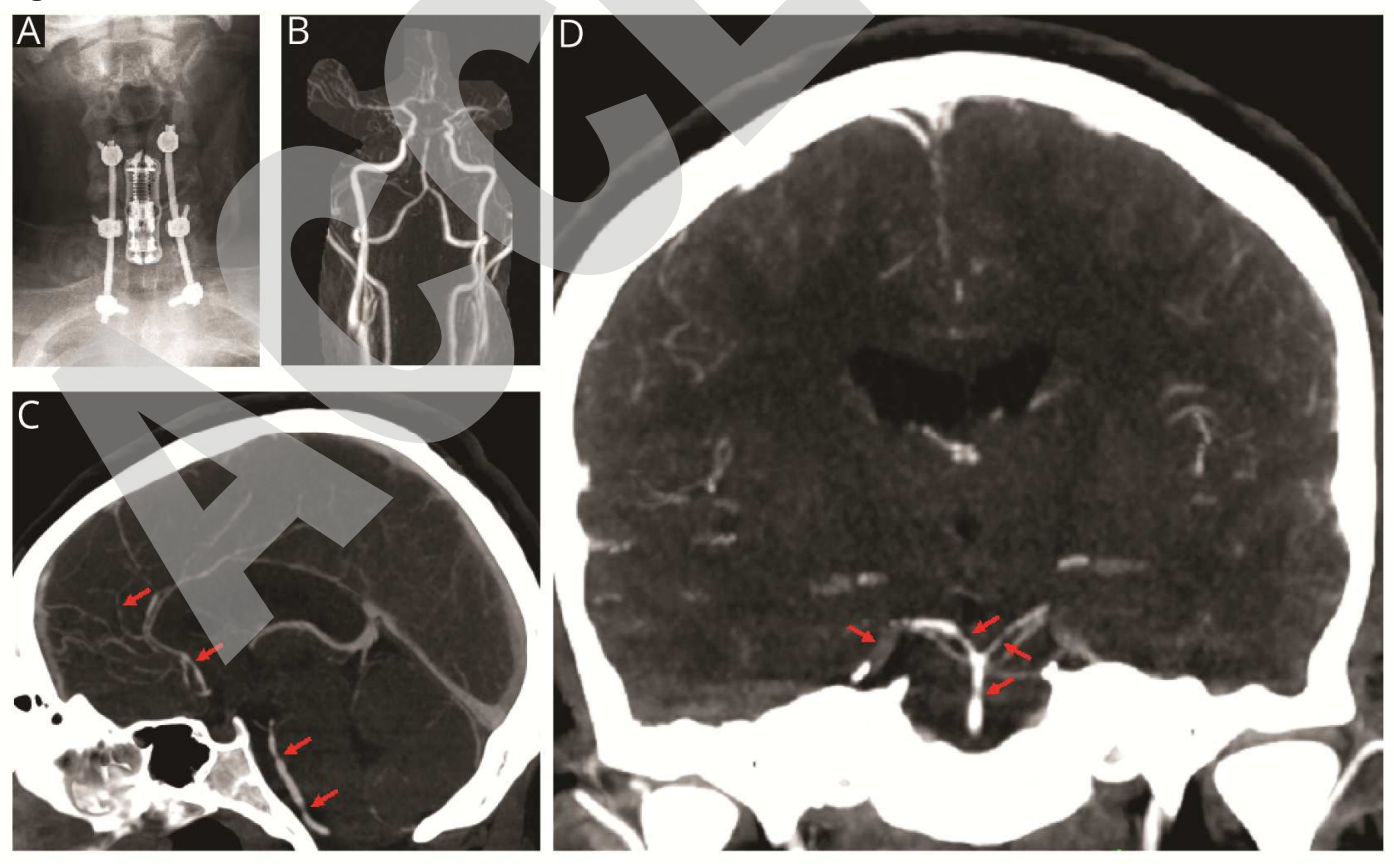
Figure 2:

MRI brain images illustrate evolution of the patient's clinical course. Panels $A$ and $B$ were obtained upon admission; Diffusion weighted imaging (DWI) (A) and apparent diffusion coefficient $(A D C)$ map $(B)$ demonstrated a left parieto-occipital lobe acute infarct. Panels $C$ and $D$ were completed upon acute worsening of headache and vision loss, with DWI (C) and ADC map (D) showing additional ischemic infarcts.

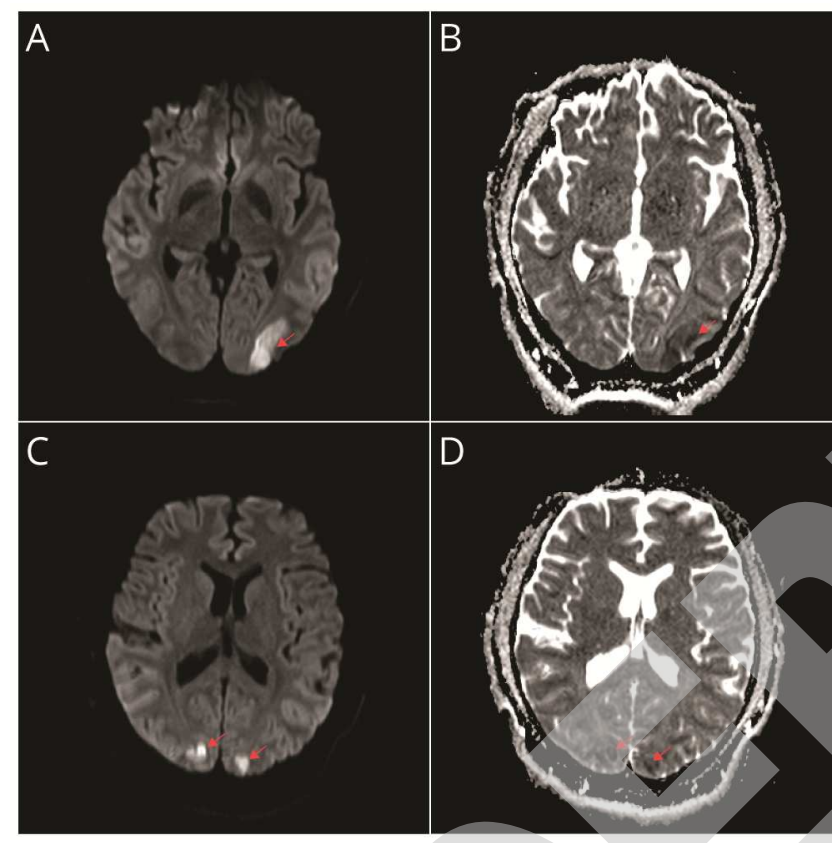




\section{Neurology}

\section{Teaching NeuroImage: Neurovascular Consequences of Autonomic Dysreflexia \\ Christina M Lineback, Eric W Moffet and Minjee Kim \\ Neurology published online October 21, 2021 \\ DOI 10.1212/WNL.0000000000013011}

\section{This information is current as of October 21, 2021}

\section{Updated Information \& Services}

\section{Subspecialty Collections}

Permissions \& Licensing

Reprints including high resolution figures, can be found at:

http://n.neurology.org/content/early/2021/10/20/WNL.0000000000013011. citation.full

This article, along with others on similar topics, appears in the following collection(s):

Autonomic diseases

http://n.neurology.org/cgi/collection/autonomic_diseases

CT

http://n.neurology.org/cgi/collection/ct

Spinal cord trauma

http://n.neurology.org/cgi/collection/spinal_cord_trauma

Spinal cord trauma; see Trauma/spinal cord trauma

http://n.neurology.org/cgi/collection/spinal_cord_trauma-see_trauma-spinal cord_trauma

Stroke in young adults

http://n.neurology.org/cgi/collection/stroke_in_young_adults

Information about reproducing this article in parts (figures,tables) or in its entirety can be found online at:

http://www.neurology.org/about/about_the_journal\#permissions

Information about ordering reprints can be found online:

http://n.neurology.org/subscribers/advertise

Neurology ${ }^{\circledR}$ is the official journal of the American Academy of Neurology. Published continuously since 1951, it is now a weekly with 48 issues per year. Copyright (C) 2021 American Academy of Neurology. All rights reserved. Print ISSN: 0028-3878. Online ISSN: 1526-632X.

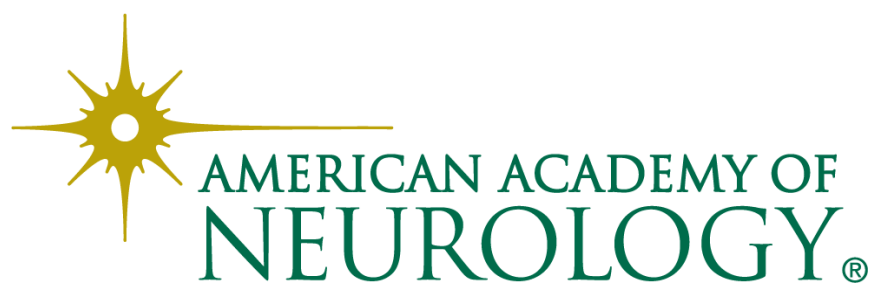

